\title{
Conducta ante los estados hipertensivos del embarazo
}

\section{Management of hypertension in pregnancy}

\author{
M. Huarte, A. Modroño, C. Larrañaga
}

\section{RESUMEN}

Los estados hipertensivos del embarazo son un conjunto de desórdenes que acontecen durante la gestación cuyo nexo común es la hipertensión. Deben ser destacados dada su implicación en la morbimortalidad materna y neonatal.

Se realiza una clasificación de los diferentes estados hipertensivos poniendo especial interés en la preeclampsia. En el texto se definen los síntomas y signos de la enfermedad y se realiza un diagnóstico diferencial entre enfermedades que debemos descartar. Es de interés identificar las gestantes con preeclampsia y todavía resulta de mayor interés descartar en ellas algún criterio de gravedad ya que esto nos obligará a realizar un manejo diferente.

A lo largo del texto, se adjuntan las indicaciones y el momento de finalización de la gestación. De igual forma se detallan los controles que deben realizarse si se opta por una conducta expectante en beneficio del prematuro. Se detallan las diferentes opciones terapeúticas antihipertensivas así como el tratamiento profiláctico de la eclampsia con sulfato de magnesio.

Destacamos por su interés el Síndrome de HELLP y la eclampsia como complicaciones. Se describe el tratamiento y la conducta que se debe tomar en la gestación.

Palabras clave. Preeclampsia. Eclampsia. Síndrome de HELLP. Hipertensión. Ptoteinuria.

\begin{abstract}
Hypertensive states of pregnancy are a set of disorders that occur during gestation whose common nexus is hypertension. They must be given special emphasis due to their implication in maternal and neonatal morbidity and mortality.

A classification is made of the different hypertensive states, with special emphasis placed on preeclampsia. This article defines the symptoms and signs of the disease and a differential diagnosis is made amongst diseases that must be ruled out. It is important to identify expectant mothers with preeclampsia, and it is of even greater importance in such cases to rule out some criterion of seriousness, as this will enable a different management to be carried out.

The article includes the indications and the moment when the pregnancy finalises. Similarly, it details the controls that must be made if an expectant management is chosen for the benefit of the premature baby. The different anti-hypertensive therapeutical options are detailed, as well as the prophylactic treatment of eclampsia with magnesium sulphate.

Because of their intrinsic interest, we draw special attention to the HELLP syndrome and to eclampsia as complications. The treatment and conduct that must be followed in gestation is described.
\end{abstract}

Key words. Pre-eclampsia. Eclampsia. HELLP Syndrome. Hypertension. Proteinuria.
Servicio de Obstetricia y Ginecología. Hospital Virgen del Camino. Pamplona.

\section{Correspondencia:}

María Huarte Ciganda

Pintor Maeztu, 10-5ํㅡ

31008 Pamplona

Tfno. 696191046

E-mail: m_huartecig@hotmail.com 


\section{INTRODUCCIÓN}

Preeclampsia se define como la aparición de hipertensión y proteinuria después de la semana 20 en una mujer embarazada previamente normotensiva. La preeclampsia severa y la eclampsia son poco frecuentes pero complican muy seriamente la gestación.

El parto y alumbramiento son el único tratamiento curativo. Se trata de una enfermedad progresiva y el parto se realiza en beneficio materno.

La razón de retrasar el parto es reducir la morbimortalidad perinatal gracias a una mayor edad gestacional y en menor grado conseguir un cerviz más favorable.

El riesgo de continuar con la gestación ocasiona un posible daño severo multiorgánico sobre cerebro, hígado, riñones, placenta/feto y sistema vascular.

\section{DEFINICIÓN Y CLASIFICACIÓN}

Los estados hipertensivos del embarazo (EHE) constituyen un conjunto de desórdenes que acontecen durante la gestación cuyo nexo común es la presencia de hipertensión arterial. Pueden ser causa de secuelas permanentes (alteraciones neurológicas, hepáticas, renales o hematológicas) e incrementan la mortalidad neonatal, asociándose con crecimiento intrauterino retardado (CIUR), oligoamnios y prematuridad. Los EHE pueden clasificarse en varios tipos ${ }^{1}$ :

1. HTA crónica: HTA previa a la gestación, diagnosticada antes de la semana 20 ó tras la semana 20 , persistiendo más allá de la semana 12 de puerperio (3\% gestaciones).

2. Hipertensión gestacional: HTA posterior a la semana 20 sin proteinuria; retorna a cifras normales antes de la semana 12 de puerperio. Puede evolucionar a preeclampsia al aparecer posteriormente proteinuria (6\% gestaciones).

3. Preeclampsia/eclampsia: HTA (TA sistólica $\geq 140 \mathrm{y} / \mathrm{o}$ TA diastólica $\geq 90$ en dos ocasiones separadas $\geq 4-6$ horas) asociada a proteinuria ( $\geq 300 \mathrm{mg}$ en orina de 24 horas $\mathrm{o} \geq 2+$ en labstics en dos ocasiones separadas $\geq 4$ horas) en gestante de más de 20 semanas previamente normotensa (3-14\% gestaciones).

A su vez se puede diferenciar entre preeclampsia leve (siendo ésta la definida previamente) y grave (presencia de uno o más de los criterios siguientes). No existe la preeclampsia moderada. La preeclampsia grave presenta uno o más de los siguientes criterios:

- TA sistólica $\geq 160 \mathrm{mmHg}$ y/o diastólica $\geq 110$ en dos determinaciones separadas en 4-6 horas, antes del inicio del tratamiento antihipertensor.

- Renales: Proteinuria $\geq 2 \mathrm{~g}$ en orina de 24 horas, oliguria $<500 \mathrm{ml} / 24$ horas, creatinina sérica $>1,2 \mathrm{mg} / \mathrm{dl}$.

- Alteraciones analíticas: pruebas funcionales hepáticas; hematológicas: trombocitopenia $(<100.000 \mathrm{~mm} 3)$, CID, hemólisis (esquistocitos en sangre periférica, $\mathrm{LDH} \geq 600 \mathrm{u} / \mathrm{l}$, haptoglobina $<0,8 \mathrm{mg} / \mathrm{dL}$ ).

- Alteraciones clínicas: cerebrales o visuales (hiperreflexia con clonus, cefalea severa, escotomas, visión borrosa, amaurosis, hemorragia retiniana, papiledema); síntomas de distensión de la cápsula hepática, dolor epigástrico o en hipocondrio derecho, nauseas, vómitos; edema agudo de pulmón.

- Manifestaciones fetales (CIUR).

La eclampsia es la aparición de convulsiones tónico-clónicas en el contexto de una paciente preeclámptica que no pueden ser atribuidas a otras causas. El $20 \%$ de las pacientes con eclampsia no presenta HTA objetiva.

4. Preeclampsia sobreañadida a HTA crónica: desarrollo de proteinuria en gestante con HTA previa conocida o empeoramiento de las cifras de ésta o de las cifras tensionales tras la semana 20.

\section{PREDICCIÓN Y PROFILAXIS DE LA PREECLAMPSIA}

Por el momento no existen medidas profilácticas efectivas de prevención primaria por desconocer su etiopatogenia (Fig. 1). Por tanto, la actuación se centra en la identificación de gestantes de riesgo para ajustar correctamente el adecuado control antenatal. El control materno per- 
sigue dos fines, el diagnóstico precoz de la preeclampsia en los casos de hipertensión gestacional y la valoración de la progresión del proceso ${ }^{2,3}$.

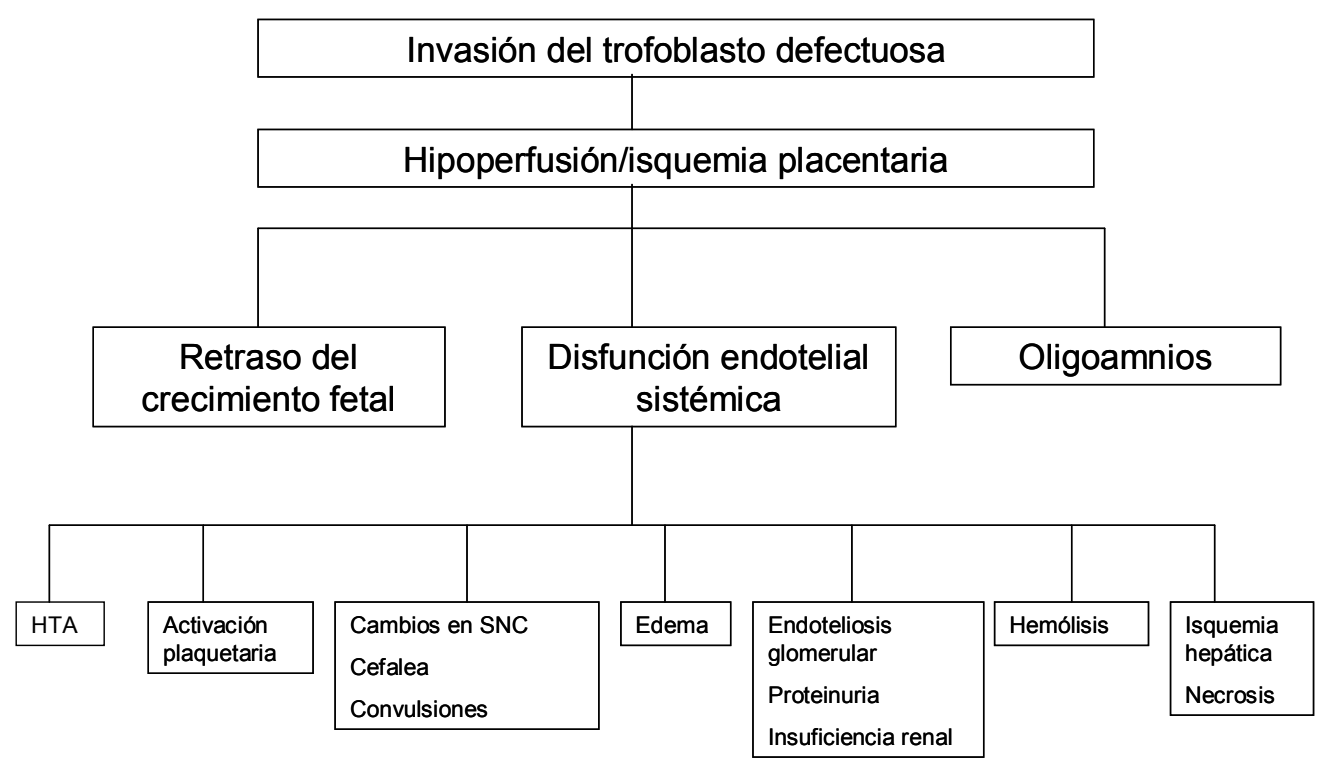

Figura 1. Fisiopatología de la preeclampsia.

\section{CONDUCTA OBSTÉTRICA DE URGENCIA}

Desde el punto de vista de la urgencia obstétrica es preciso distinguir los cuadros clínicos según su presentación:

- Gestantes hipertensas asintomáticas o paucisintomáticas en las que se deberá establecer el tipo de cuadro EHE y su gravedad.

- Gestantes con sintomatología que requieran diagnóstico diferencial entre EHE y otros cuadros clínicos distintos.

En la atención a estas gestantes se iniciará la valoración materna y fetal de forma conjunta, que permitirá establecer el diagnóstico, su gravedad y su posible repercusión fetal ${ }^{4,5}$.

- Control materno: monitorización de la tensión arterial cada 15 minutos. Toma de muestra de orina para estu- dio semicuantitativo de la proteinuria (la presencia de $2+$ se establece como positivo para proteinuria) y en caso de positividad o duda se iniciará recogida de orina para valoración de diuresis, cuantificación de proteinuria de 24 horas y aclaramiento de creatinina. Solicitud de analítica urgente con hemograma completo, pruebas de coagulación, bioquímica (transaminasas, creatinina, ácido úrico, LDH, bilirrubina). Valoración clínica de la gestante con anamnesis

- Control fetal: en gestaciones por encima de la semana 28 , monitorización con cardiotocografía. Si es claramente patológica podría prescindirse de evaluación Doppler. Ecografía fetal: determinación de la estática fetal, estimación del peso fetal, valoración del índice de líquido amniótico (ILA). Si puede realizarse, la medición Doppler de la arteria umbilical y la cerebral 
media aportan datos para la valoración fetal

El establecimiento del juicio clínico requiere un diagnóstico diferencial previo que se establecerá con distintas entidades según signos y síntomas teniendo en cuenta los factores de riesgo de la enfermedad (nuliparidad, gestación múltiple, enfermedad trofoblástica, obesidad, antecedentes familiares, personales de preeclampsia, HTA crónica, enfermedad renal crónica, diabetes mellitus pregestacional, trombofilia, exposición limitada al esperma de la pareja).

- HTA: de nueva aparición en la segunda mitad de la gestación inicialmente se diagnostica como preeclampsia hasta que no se pueda identificar otra causa. La posibilidad de una enfermedad microvascular subyacente asociada es más factible cuando la aparición del cuadro se establece en la primera mitad de la gestación, o no se cumple la primipaternidad o la instauración de la misma es precoz y grave desde el inicio.

- Dolor torácico: está más relacionado con edema pulmonar o fallo cardiaco congestivo que con infarto de miocardio.

- Disnea: la asociación más frecuente es con edema pulmonar. Suele evolucionar en horas. El establecimiento del edema pulmonar es más probable que se establezca en el puerperio, de ahí la importancia de un buen control del equilibrio de entradas y salidas de fluidos y de los sangrados.

- Tos: síntoma de presentación frecuente del edema de pulmón. Descartar la presencia de fiebre y establecer diagnóstico diferencial con neumonía. En pacientes apiréticas descartar que esté relacionado con reflujo gastroesofágico o asma.

- Taquipnea: una frecuencia respiratoria superior a 25 inspiraciones por minuto requiere estudio, siendo la primera causa el edema pulmonar, pero se debe descartar una complicación tromboembólica.

- Edema: ya no es criterio diagnóstico de preeclampsia por su frecuente aparición en la gestación. En la evalua- ción de las pacientes hipertensas tiene valor la presencia de edema facial, especialmente palpebral, dado que no es habitual en las gestantes.

- Náuseas y/o vómitos: su establecimiento más allá del primer trimestre inicialmente no se debe etiquetar como hiperemesis gravídica. Las entidades por descartar serán intraabdominales -las más frecuentes-, intracraneales y metabólicas. Tiene interés destacar que deben inspeccionarse las características del vómito (alimentos no digeridos, bilis, sangre, fecaloideos...) pues orientan a distintos procesos.

- Intraabdominales: colelitiasis y/o colecistitis (asocia dolor que se exacerba tras las ingestas), obstrucción intestinal, pancreatitis.

- Intracraneales: hipertensión intracraneal (realizar fondo de ojo para descartar papiledema).

- Metabólicas: intoxicaciones varias (destacar el sulfato de magnesio), hiperglucemia, hiperamonemia.

- Autoprovocados.

- Dolor abdominal: el dolor característico de la preeclampsia es el localizado en epigastrio y/o en el cuadrante superior derecho, que no se modifica con la ingesta de alimentos, ni irradia. El dolor dependiente de una víscera sin implicación del peritoneo no se modifica por el embarazo, su clínica será similar a la que presente en pacientes no gestantes. Tiene interés evaluar el comportamiento de la gestante ya que los dolores de víscera hueca de características cólicas no se afectan por el movimiento, de manera que las pacientes suelen estar agitadas. Por el contrario, si el origen álgico es peritoneal éste se agrava con el movimiento, hecho que condiciona que la paciente esté en reposo.

- El dolor biliar se relaciona con ingesta, irradia a dorso y generalmente asocia fiebre.

- El dolor de la obstrucción de intestino delgado suele localizarse periumbilical y la de intestino grueso en hipogastrio. 
- Las molestias asociadas a las hepatitis se acompañan casi constantemente con icteria y en ausencia de HTA y proteinuria.

- El desprendimiento precoz de placenta normoinserta (DPPNI) suele ser un dolor focalizado sobre el útero y su incidencia aumenta en el contexto de la preeclampsia.

- Convulsiones: las convulsiones eclámpticas no suelen asociar aura, ni focalización posterior (excepto si asocian complicación hemorrágica) y se caracterizan por ser autolimitadas características que permiten el diagnóstico diferencial con las convulsiones tipo gran mal de la epilepsia.
- La clínica derivada de la trombosis venosa cerebral, de las vasculitis y el lupus eritematoso sistémico mimetiza mucho a la eclampsia.

- En ausencia de HTA y/o proteinuria descartar: traumatismo cerebralhemorragia, infección SNC, intoxicación (cocaína, anfetaminas...), metabolopatías.

Por otro lado se destacarán los cuadros clínicos más importantes en el diagnóstico diferencial de las formas graves de preeclampsia y/o síndrome de HELLP con la púrpura trombótica trombocitopéniaca (PTT), síndrome hemolítico urémico (SHU) y el Hígado Graso Agudo del Embarazo (HGAE) (Tabla 1).

Tabla 1. Diagnóstico diferencial del Síndrome. Hellp.

\begin{tabular}{lcccc}
\hline \multicolumn{1}{c}{ Alteración } & HELLP & $\begin{array}{c}\text { Púrpura } \\
\text { trombocitopénica }\end{array}$ & $\begin{array}{c}\text { Síndrome } \\
\text { hemolítico } \\
\text { urémico }\end{array}$ & $\begin{array}{c}\text { Hígado agudo } \\
\text { graso del } \\
\text { embarazo }\end{array}$ \\
\hline HTA & $75 \%$ & Raro & Presente & $25-50 \%$ \\
\hline Proteinuria & $90 \%$ & Variable & Presente & Variable \\
\hline Trombocitopenia & $100 \%$ & $100 \%$ & $50 \%$ (inicio) & Variable \\
\hline LDH & $\uparrow$ & $\uparrow$ & $\uparrow \uparrow$ & $\uparrow$ \\
\hline Tiempos coagulación & - & - & - & $\uparrow$ \\
\hline Fibrinógeno & - & - & - & $\downarrow$ \\
\hline PDF & - & - & $\uparrow$ & $\uparrow$ \\
\hline Antitrombina III & $\downarrow$ & - & - & $\downarrow$ \\
\hline Bilirrubina & $\uparrow$ & $\uparrow$ & $\uparrow$ & $\uparrow \uparrow$ \\
\hline Amoniaco & - & - & - & $\downarrow \uparrow$ \\
\hline Glucemia & - & - & - & $\uparrow$ \\
\hline Anomalías renales & $\uparrow$ & - & - & - \\
\hline Anomalías neurológicas & $\uparrow$ & $\uparrow \uparrow$ & $\uparrow$ & - \\
\hline Fiebre & - & $\uparrow$ & $\uparrow$ & \\
\hline
\end{tabular}

LDH: Lactato deshidrogenada.

PDF: Productos de degradación del fibrinógeno.

\section{CONDUCTA Y TRATAMIENTO}

El principal objetivo del tratamiento de la preeclampsia es el bienestar materno. En ocasiones la finalización de la gestación conlleva un riesgo fetal elevado por la pre- maturidad yatrógena, por este motivo el tratamiento de estas pacientes dependerá de la edad gestacional y del bienestar materno y fetal (Fig. 2). 


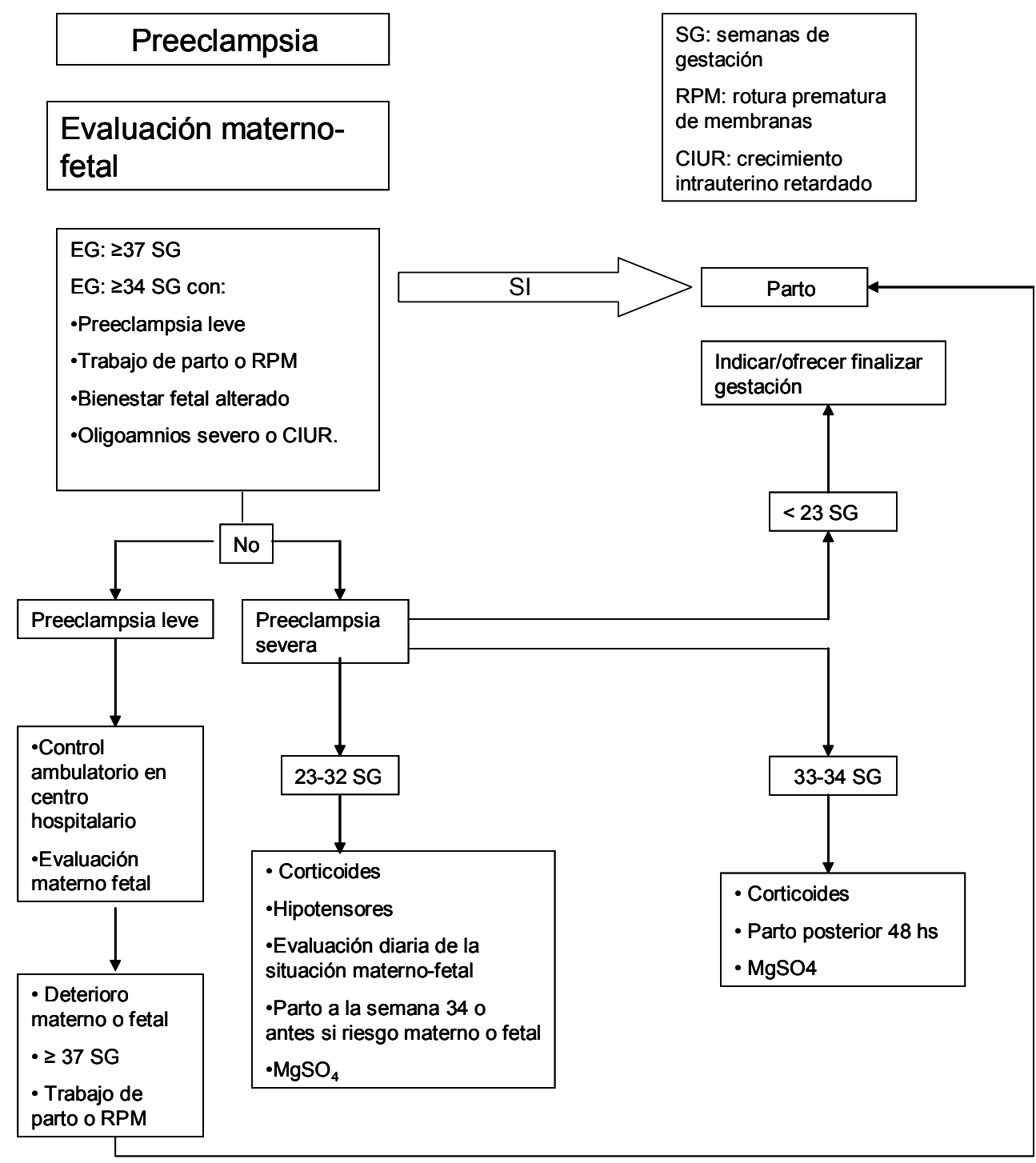

Figura 2. Conducta en la preeclampsia (Modificado de Sibai y $\operatorname{col}^{6}$ ).

Por tanto, en el momento de establecer la conducta obstétrica conocer con exactitud la edad gestacional es una posiblemente la información más determinante para el clínico.

\section{Finalización de la gestación}

- Gestación a término >37 semanas, no debiendo superar la semana 40 inclu- so en presencia de condiciones cervicales desfavorables.

- Preeclampsia grave en gestante de $>34$ semanas.

- Por debajo de la semana 34 ante la presencia de:

- Pródromos de eclampsia o eclampsia.

- HTA severa resistente al tratamiento (dos hipotensores a dosis máximas de tratamiento). 
- Fallo multiorgánico (deterioro de la función renal u oligoanuria, de la función hepática, trombocitopenia progresiva).

- Hemorragia cerebral, EAP, rotura hepática, DPPNI.

- Síndrome de HELLP no estable.

- Pérdida del bienestar fetal (estudio de Eco-Doppler de arteria uterina con diástole ausente o revertida, registro cardiotocográfico -CTG- no tranquilizador, perfil biofísico $<4$ ).

\section{CONDUCTA EXPECTANTE DE LA PREECLAMPSIA LEVE}

No es necesario el ingreso si de forma ambulatoria se consigue un control adecuado. Las medidas a tener en cuenta son $^{7}$ :

- Control de TA cada 12 horas.

- Proteinuria cualitativa/24 horas y en orina de 24 horas (1-2 veces/sem).

Tabla 2. Fármacos antihipertensivos utilizados en el manejo de los estados hipertensivos del embarazo.

\begin{tabular}{|c|c|c|c|c|c|}
\hline Fármaco & Nombre comercial & Posología & Dosis máxima & Efectos secundarios & Contraindicaciones \\
\hline Labetalol & $\begin{array}{l}\text { Trandate }{ }^{\circledR} \\
\text { (cp de } 100-200 \\
\text { mg) } \\
\text { (ampollas de } 100 \\
\text { mg) }\end{array}$ & $\begin{array}{l}\text { vo: } 50-400 \mathrm{mg} / 6 \mathrm{~h} \\
\text { iv: bolus inicial } \\
20 \mathrm{mg} \text { (repetir } \\
40,60,80 \mathrm{mg} \text { ) } \\
\text { bolus inicial de } \\
50 \mathrm{mg} \text { (repetir } 4 \\
\text { bolus de } 50 \mathrm{mg} \text { ) } \\
\text { perfusión: } 50-400 \\
\text { mg/ } 6 \mathrm{~h}\end{array}$ & $2400 \mathrm{mg} / 24 \mathrm{~h}$ & Bradicardia & $\begin{array}{l}\text { Insuficiencia } \\
\text { cardiaca } \\
\text { Asma bronquial } \\
\text { Bradicardia } \\
\text { materna }\end{array}$ \\
\hline Hidralazina & $\begin{array}{l}\text { Hydrapress }{ }^{\circledR} \\
\text { (cp de } 25,50 \mathrm{mg} \text { ) } \\
\text { (ampollas de } 1 \\
\text { mg) }\end{array}$ & $\begin{array}{l}\text { vo: } 25-50 \mathrm{mg} / 6-8 \mathrm{~h} \\
\text { iv:bolus inicial } 10 \\
\text { mg (repetir } 4 \\
\text { bolus de } 5 \mathrm{mg} \text { ) } \\
\text { perfusión } 3-7 \mathrm{mg} / \\
\mathrm{kg} / \mathrm{h}\end{array}$ & $200 \mathrm{mg} / 24 \mathrm{~h}$ & Taquicardia & $\begin{array}{c}\text { Enfermedad } \\
\text { coronaria } \\
\text { Cardiopatía } \\
\text { Taquicardia }\end{array}$ \\
\hline Nifedipino & $\begin{array}{l}\text { Adalat }{ }^{\circledR} \\
\text { (cp de } 10 \mathrm{mg} \text { ) } \\
\text { Adalat Retard® } \\
\text { (cp de } 20 \mathrm{mg} \text { ) } \\
\text { Adalat } 0 \text { ros } ® \\
\text { (cp de } 30 \text { y } 60 \mathrm{mg} \text { ) }\end{array}$ & vo: $10-20 \mathrm{mg} / 6-8 \mathrm{~h}$ & $60-80 \mathrm{mg} / 24 \mathrm{~h}$ & $\begin{array}{l}\text { Cefalea } \\
\text { Rubor } \\
\text { Taquicardia }\end{array}$ & $\begin{array}{l}\text { Estenosis } \\
\text { intestinal }\end{array}$ \\
\hline $\begin{array}{l}\text { Nitroprusiato } \\
\text { sódico iv }\end{array}$ & & $\begin{array}{l}\text { Perfusión continua } \\
\text { a dosis de } 0,25 \mu \mathrm{g} / \\
\mathrm{kg} / \mathrm{min} \text { aumentando } \\
\mathrm{la} \text { dosis } 0,25 \mu \mathrm{g} / \mathrm{kg} / \\
\text { min cada } 5 \mathrm{~min} \text {. }\end{array}$ & $\begin{array}{l}\text { No administrar } \\
>4 \text { horas con feto } \\
\text { intraútero. }\end{array}$ & $\begin{array}{l}\text { Muy fetotóxico } \\
\text { por acúmulo de } \\
\text { cianida }\end{array}$ & \\
\hline Nitroglicerina iv & & $\begin{array}{l}5 \mu \mathrm{g} / \mathrm{min} \mathrm{v} \\
\text { doblando la dosis } \\
\text { cada } 5 \text { min }\end{array}$ & $100 \mu \mathrm{g} / \mathrm{min}$ & & $\begin{array}{l}\text { Encefalopatía } \\
\text { hipertensiva }\end{array}$ \\
\hline Alfa metildopa & Aldomet ${ }^{\circledR}$ & $250-500 \mathrm{mg} / 8$ h v.o. & & & \\
\hline Furosemida & Seguril® & $\begin{array}{l}20-40 \text { mg iv } \\
\text { en bolo o en } \\
\text { infusión sin diluir } \\
\text { (individualizar } \\
\text { dosis en función de } \\
\text { la dosis o mejora } \\
\text { hemodinámica) }\end{array}$ & $\begin{array}{l}\text { Sólo indicado en } \\
\text { caso de oliguria, } \\
\text { preeclampsia } \\
\text { sobreañadida } \\
\text { a cardiopatía } \\
\text { o neuropatía, } \\
\text { edema cerebral o } \\
\text { pulmonar }\end{array}$ & $\begin{array}{l}\text { Disminuye } \\
\text { el volumen } \\
\text { plasmático } \\
\text { y el flujo } \\
\text { uteroplacentario }\end{array}$ & $\begin{array}{l}\text { IRanúrica } \\
\text { Hipopotasemia } \\
\text { Hiponatremia } \\
\text { Hipovolemia } \\
\text { Coma hepático }\end{array}$ \\
\hline
\end{tabular}

Contraindicados el atenolol que se ha asociado con crecimiento intrauterino retardado (CIUR) y alteraciones en el CTG, los IECAS que se asocian a pérdidas fetales precoces y tardías, insuficiencia renal y malformaciones fetales. (nivel de evidencia II 
- Analítica: hemograma, transaminasas, LDH, creatinina, ácido úrico, urea, pruebas de coagulación y de productos de degradación del fibrinógeno bisemanales.

- Control de movimientos fetales, durante 1 hora/ día si $<6$ movimientos acudir al hospital.

- Maduración pulmonar si EG <34 semanas con betametasona $12 \mathrm{mg} / 24 \mathrm{~h}$ dos dosis.

- CTG cada 7-15 días desde la semana 28 y semanal a partir de la 34 (nivel de evidencia II).

- Eco Doppler (biometría e índice de líquido amniótico cada 7-15 días, flujometría cada 7-15 días; o antes si alteraciones en arteria umbilical, cerebral media o ductus venoso).

\section{Tratamiento}

1. Reposo: no ha demostrado que mejore el pronóstico. Puede mejorar la retención hídrica y la perfusión fetal.

2. Dieta normocalórica, normoproteica y normosódica.

3. Obtener TA entre 140-145 de sistólica y 90-95 de diastólica (Tabla 2). (No indicado tratamiento hipotensor si TA $<160 / 110 \mathrm{~mm} \mathrm{Hg}$, no mejora el pronóstico y puede empeorar la situación fetal disminuyendo el flujo placentario. Ningún fármaco previene la evolución a preeclampsia grave. El manejo expectante permite valorar la progresión de la enfermedad).

\section{CONDUCTA EXPECTANTE DE LA PREECLAMPSIA GRAVE}

El tratamiento de la preeclampsia grave es la finalización de la gestación en la semana 34 (nivel de evidencia IV), en gestaciones $<34$ semanas puede prolongarse con conducta expectante ya que puede mejorar el pronóstico neonatal sin empeorar el pronóstico materno (nivel de evidencia $\mathrm{I}^{7-9}$.

- Ingreso hospitalario en centro de nivel terciario.

- Control de la TA. Control de ésta cada 5 min hasta la estabilización del cua- dro. Posteriormente cada 30 minutos.

- Exploración general: nivel de conciencia, presencia de localidad neurológica, alteraciones del fondo de ojo, signos de edema pulmonar o anomalías cardiacas, dolor en epigastrio, hiperreflexia, grado de edema, petequias, puntos sangrantes...

- Realizar tacto vaginal con el fin de valorar una posible inducción de parto.

- Control analítico, con hemograma, función hepática y renal, pruebas de coagulación cada 4-6 horas según evolución.

- Proteinuria cualitativa diaria y en orina de 24 horas bisemanal (nivel de evidencia III).

- Sonda de Foley permanente para control de diuresis horaria (debe ser $>30$ $\mathrm{ml} /$ hora) y balance hídrico.

- Fluidoterapia anteparto, solución de cristaloides (fisiológico o Ringer Lactato) a un ritmo de $60-125 \mathrm{ml} /$ hora, a menos que existan pérdidas extras de líquidos (vómitos, diarrea...). En caso de terapia hipotensora o de anestesia epidural administrar adicionalmente 1.000-1.500 ml de la misma solución a razón de $500 \mathrm{ml}$ cada 30 minutos. Objetivo conseguir flujo urinario $\geq 30 \mathrm{ml} /$ hora.

- CTG basal al menos dos veces al día por encima de la semana 26-28 (nivel evidencia I).

- Ecografia fetal: crecimiento fetal (cada 15 días), líquido amniótico y flujometría (cada 48 horas).

- Maduración pulmonar entre las semanas 24-34 de gestación. Administrar betametasona a $12 \mathrm{mg} / 24$ horas/2 días IM (nivel de recomendación I).

- Control de presión venosa central si existe edema agudo de pulmón, oliguria persistente, insuficiencia cardiaca, hipertensión severa refractaria.

- Tratamiento hipertensivo. Mantener TA $<160 / 100$ y $\geq 140 / 90$. El fármaco debe ser potente, seguro, de rápida actuación, controlable y sin efectos secundarios maternos ni fetales. El tratamiento hipotensor previene las complicaciones cerebrovasculares y cardiovasculares maternas. Se descri- 
ten los antihipertensivos de elección en la tabla 2. Contraindicados atenolol, IECAS y ARA 2 (nivel de recomendación II).

- Tratamiento profiláctico de la convulsiones con sulfato de magnesio (nivel de evidencia I). No evita el desarrollo de otras complicaciones como EAP, accidente cerebro-vascular, hematoma hepático o insuficiencia renal.

No es preciso realizar magnesemias para evitar los efectos adversos por sulfato de magnesio; la valoración clínica de los signos de intoxicación puede ser suficiente para su control ${ }^{10,11}$. Los signos por controlar son: el reflejo patelar debe estar presente (si se administra anestesia epidural evaluar reflejo cubital), frecuencia respiratoria mayor 12 inspiraciones por minuto, diuresis superior a $25-30 \mathrm{ml} /$ hora, control de saturación $\mathrm{O}_{2}$.

Se recomiendo su uso durante el parto y en las primeras 24-48 horas en las paciente con preeclampsia grave y en preeclampsia con signos de afectación neurológica.

Si no se finaliza la gestación a corto plazo, interrumpir el sulfato de magnesio cuando se prevé la continuación de la gestación, reiniciando con signos de deterioro materno o si se prevé la finalización de la gestación en 24-48 horas.

Las alternativas para la prevención de la eclampsia están descritas en la tabla 3.

Tabla 3. Profilaxis y tratamiento de las convulsiones eclámpticas.

\begin{tabular}{|c|c|c|c|c|c|c|}
\hline & \multicolumn{2}{|c|}{ Preclampsia } & \multicolumn{2}{|c|}{ Eclampsia } & \multirow[b]{2}{*}{ Contraindicaciones } & \multirow[b]{2}{*}{ Antídoto } \\
\hline & $\begin{array}{l}\text { Dosis de } \\
\text { ataque }\end{array}$ & $\begin{array}{c}\text { Dosis de } \\
\text { mantenimiento }\end{array}$ & $\begin{array}{l}\text { Dosis de } \\
\text { ataque }\end{array}$ & $\begin{array}{c}\text { Dosis de } \\
\text { mantenimiento }\end{array}$ & & \\
\hline $\mathrm{MgSO}_{4}$ & $\begin{array}{l}2-4 \mathrm{~g} \text { iv a pasar } \\
\text { en } 5-10 \mathrm{~min}\end{array}$ & $\begin{array}{l}1,5 \mathrm{~g} / \text { hora iv } \\
\text { en perfusión } \\
\text { continua }\end{array}$ & $\begin{array}{l}4-6 \text { gr ev en } \\
5-10 \text { minutos }\end{array}$ & $\begin{array}{l}2 \mathrm{gr} / \text { hora en } \\
\text { perfusión } \\
\text { continua; si } \\
\text { recurrencia de } \\
\text { las convulsiones } \\
\text { nuevo bolo de } \\
2 \text { g o aumentar } \\
\text { el ritmo de la } \\
\text { infusión. }\end{array}$ & $\begin{array}{l}\text { Miastenia gravis } \\
\text { Infarto de } \\
\text { miocardio } \\
\text { reciente } \\
\text { Bloqueo cardiaco } \\
\text { Afectación renal }\end{array}$ & $\begin{array}{l}\text { Gluconato cálcico } \\
1 \text { g iv a pasar en } \\
3-4 \text { min ( } 10 \text { ml de } \\
\text { gluconato cálcico } \\
\text { al } 10 \%)\end{array}$ \\
\hline Fenitoína & $\begin{array}{l}10-15 \mathrm{mg} / \mathrm{kg} \text { iv } \\
\text { lentamente }\end{array}$ & $\begin{array}{l}200 \mathrm{mg} / 8 \mathrm{hs} \text { iv } \\
\text { o vo }\end{array}$ & $\begin{array}{l}15 \mathrm{mg} / \mathrm{Kg} \text { iv } \\
\text { a pasar en } 1 \\
\text { hora }\end{array}$ & $\begin{array}{l}250-500 \mathrm{mg} / 12 \mathrm{~h} \\
\text { oral o iv }\end{array}$ & $\begin{array}{l}\text { Porfiria aguda } \\
\text { intermitente } \\
\text { Bradicardia } \\
\text { Alteraciones de la } \\
\text { conducta }\end{array}$ & \\
\hline Diazepam & $\begin{array}{l}10-20 \mathrm{mg} \text { iv en } \\
5 \mathrm{~min}\end{array}$ & $\begin{array}{l}\text { Perfusión hasta } \\
\text { un máx de } 100 \\
\text { mg/ } 24 \text { h }\end{array}$ & $40 \mathrm{mg}$ iv & $\begin{array}{l}10 \mathrm{mg} / \mathrm{h} \text { en } \\
\text { perfusión } \\
\text { continua }\end{array}$ & $\begin{array}{l}\text { Glaucoma de } \\
\text { ángulo cerrado } \\
\text { Hipercapnia crónica } \\
\text { grave } \\
\text { Enf. musculares } \\
\text { (miastenia gravis) } \\
\text { EPOC } \\
\text { Insuficiencia } \\
\text { hepática }\end{array}$ & $\begin{array}{l}\text { Flumazenilo 0,2-0,3 } \\
\text { mg iv en } 15 \text { sg, } \\
\text { si no respuesta } \\
\text { admón. cada } 60 \\
\text { sg } 0,1 \mathrm{mg} \text { hasta } \\
\text { máximo de } 1 \mathrm{mg} .\end{array}$ \\
\hline
\end{tabular}

\section{Niveles terapéuticos:}

$\mathrm{MgSO}_{4}$ : Niveles plasmáticos entre 3,5-9,6 (realizar magnesemia o valorar signos clínicos; reflejo patelar debe estar presente, si anestesia epidural evaluar reflejo cubital, frecuencia respiratoria $>12 \mathrm{~min}$, diuresis $>25-30 \mathrm{ml} /$ hora, control de $\mathrm{Sat}_{2}$.

Fenitoina: Niveles terapéuticos entre $5-15 \mu \mathrm{g} / \mathrm{ml}$.

Diazepam: Dosis terapeúticas casi en umbral de la toxicidad. Pérdida de la variabilidad en el CTG con aumento de la morbilidad perinatal. Valorar grado de conciencia materno e intubación. 


\section{CONDUCTA Y TRATAMIENTO DE LA ECLAMPSIA}

Es necesario tratar la aparición de convulsiones en el contexto de hipertensión en el embarazo con o sin proteinuria en ausencia de otras causas conocidas de convulsiones ${ }^{8,12}$. Hacer diagnóstico diferencial con otras entidades (Tabla 4).

Tabla 4. Diagnóstico diferencial de la eclampsia

\begin{tabular}{l}
\hline Accidentes cerebrovasculares \\
Hemorragia intracerebral \\
Trombosis arterial o venosa. Embolismo \\
arterial \\
Encefalopatía hipóxico isquémica \\
Angiomas \\
\hline Enfermedades hipertensivas \\
Encefalopatía hipertensiva \\
Feocromocitoma \\
\hline Lesiones ocupantes de espacio en el SNC \\
Tumor cerebral \\
Enfermedad trofoblástica gestacional \\
metastásica \\
Absceso \\
\hline Trastornos metabólicos \\
Hipoglucemia \\
Uremia \\
Hiponatremia \\
\hline Etiología infecciosa \\
Meningitis \\
Encefalitis \\
\hline Púrpura trombocitopénica trombótica \\
Trombofilia \\
Epilepsia idiopática \\
Vasculitis cerebral \\
Síndrome pospunción dural \\
\hline
\end{tabular}

La incidencia de convulsiones depende de la severidad de la preeclampsia pero se estima $<1 \%$. El $50 \%$ de los casos debuta anteparto, el $25 \%$ en el parto y el resto en el postparto. Su etiología es desconocida, se propone la hipótesis de el vasoespasmo cerebral con isquemia local, la encefalopatía hipertensiva con hiperperfusión, el edema vasogénico y el daño endotelial.

Esta afectación es más frecuente en raza no blanca, un $16 \%$ normotensas y un $20 \%$ presenta ausencia de proteinuria. El $60-75 \%$ presentan pródromos como cefalea occipital o frontal, visión borrosa, fotofo- bia, dolor epigástrico o de hipocondrio derecho, y confusión mental.

El factor de riesgo más importante es la ausencia de tratamiento y de control médico previo. Es una urgencia vital que causa unas 50.000 muertes maternas/año en el mundo.

\section{Principios del tratamiento de una crisis de eclampsia}

1. Soporte de las funciones vitales maternas. Asegurando vía aérea (tubo de Mayo) para oxigenar a la madre y evitar lesiones, y vía venosa para administrar fármacos y obtención de analíticas.

2. Control de la hipertensión en un rango seguro con los mismos criterios que para la preeclampsia grave. Debe realizarse al mismo tiempo o incluso antes que el tratamiento anticonvulsivante. Monitorización de la TA cada 2-5 minutos.

3 . Control de las convulsiones y prevención de la recurrencias. Tratamiento anticonvulsivante (Tabla 4). Utilización de sulfato de magnesio (nivel de evidencia I). Contrario a la tendencia natural, no es conveniente y es potencialmente peligroso abolir y acortar inmediatamente la convulsión. La mayoría de las convulsiones eclámpticas se resuelven en $60-90 \mathrm{seg}$. Cuando la convulsión termina, se debe iniciar el tratamiento con sulfato de magnesio. Corrección de la hipoxemia materna y/o acidosis en caso de existir. En ausencia de respuesta usar: diazepan y fenitoína como en la preclampsia grave, barbitúricos de acción corta (tiopental o amobarbital), intubación y curarización.

4. Inicio del proceso de parto una vez estabilizada la situación. Este aspecto es de gran importancia, ya que una vez controlada o finalizada la crisis convulsiva, es aconsejable disponer de la máxima información para estabilizar al máximo a la paciente y finalizar la gestación en las mejores condiciones posibles, lo cual incluye la valoración de un traslado a otro centro si con esto se 
mejora la probabilidad de atenciones a la madre o al feto. La presencia de patrones patológicos de la FC durante la crisis convulsiva no es indicación de cesárea ya que habitualmente se recuperan a los 3-15 min. Si persisten tras la estabilización de la paciente hay que sospechar un desprendimiento de placenta o una pérdida de bienestar fetal.

5. Fluidoterapia: perfusión de mantenimiento recomendada de $85-100 \mathrm{ml} /$ hora. Administrar $500 \mathrm{ml}$ de fisiológico o Ringer Lactato antes de la anestesia locorregional o del inicio del tratamiento hipotensor.

6. Control de complicaciones: EAP, insuficiencia cardiaca, DPPNI, coagulación intravascular diseminada y síndrome de HELLP. Hacer TC o RM sólo si localidad neurológica o coma prolongado.

\section{SÍNDROME HELLP}

Acrónimo de hemólisis, elevación enzimas hepáticas y descenso de plaquetas (hemolysis, elevated liver enzymes, lower platelets). Es una variante severa de la preeclampsia descrita por Wenstein en $1982^{13}$.

$\mathrm{La}$ incidencia en mujeres con preeclampsia oscila entre el 4-12\%. En preeclampsia grave es de alrededor del 20-25\%. Se da con mayor frecuencia en raza blanca, añosas y multiparas.

\section{Etiopatogenia}

La hemólisis por anemia hemolítica microangiopática es el factor determinante (esquistocitos y reticulocitos en frotis de sangre periférica, aumento de bilirrubina y de LDH y disminución por consumo de haptoglobina).

La afectación hepática es por depósitos de material fibrinoide en el espacio parenquimatoso periportal (responsable de los síntomas clásicos de dolor epigástrico y en hipocondrio derecho y de la elevación de enzimas hepáticos).

La disminución de plaquetas es por la microangiopatía con aumento de la actividad plaquetaria.

\section{Diagnóstico}

Criterios diagnósticos por Sibai en $1990^{14}$.

1. Hemólisis: presencia de esquistocitos en sangre periférica, bilirrubina $\geq 1,2 \mathrm{mg} / \mathrm{dL} ; \mathrm{LDH} \geq 600 \mathrm{U} / \mathrm{L}$.

2. Aumento de los enzimas hepáticos: $\mathrm{AST} \geq 70$ o mayor a $3 \mathrm{DS}$.

3. Trombocitopenia:plaquetas $\leq 100.000$ cél/L. Otra clasificación intenta estratificar según la gravedad.

- Clase $1<50.000$ cél/L

- Clase $2 \leq 100.000$ cél /L

- Clase 3: 150.000-100.000 cél /L

\section{Clínica}

En un $67 \%$ de las pacientes aparecen en el comienzo del tercer trimestre (27-37 SG). El 25\% de los pacientes manifiestan la enfermedad en el postparto.

Fatiga y síntomas no específicos acontecen en el $90 \%$. Náuseas, cefalea, dolor abdominal y edema se encuentran en más de la mitad.

HTA >160/110 mmHg aparece en $2 / 3$ de los pacientes. Otras en el rango de $140-160 / 90-110$ y un 15\% diastólicas menores de 90 .

El 85\% de las pacientes presentan proteinuria de $\geq 2+$, el $9 \%$ solo $1+$ y el $6 \%$ ausencia de proteinuria.

Debemos tener en cuenta que el síndrome HELLP puede ocurrir en pacientes con PA ligeramente elevada o dentro de límites normales y ausencia de proteinuria.

\section{Tratamiento}

Una vez diagnosticado se debe considerar finalizar la gestación. Las complicaciones neonatales son las mismas que para los casos de preeclampsia grave y dependen de la edad y la administración de corticoesteroides prenatales (nivel de evidencia III). El manejo expectante ha demostrado mejorar el pronóstico neonatal sin incrementar el riesgo materno, salvo en la administración de derivados sanguíneos (nivel de evidencia III) ${ }^{15}$.

La conducta es similar a la preclampsia grave con las siguientes salvedades: el ini- 
cio del tratamiento antihipertensor con TA de 150/90 mm Hg y objetivo terapéutico de $130 / 80 \mathrm{mmHg}$ y la posible administración de corticoesteroides. La dosis es doble que la utilizada para la maduración pulmonar y la mitad de la recomendada para el tratamiento de la púrpura trombocitopénica idiopáti- ca en adultos, dexametasona $10 \mathrm{mg}$ iv/12 h y dos dosis, mínimo, adicionales en el postparto hasta alcanzar $100.000 \mathrm{plaq} / \mathrm{mm}^{3}$ seguidas de dos dosis de $5 \mathrm{mg}$ iv/12 h, susceptible de variaciones en función de las circunstancias individuales. (Fig. 3). No obstante el uso de corticoides es muy controvertido ${ }^{15-17}$.

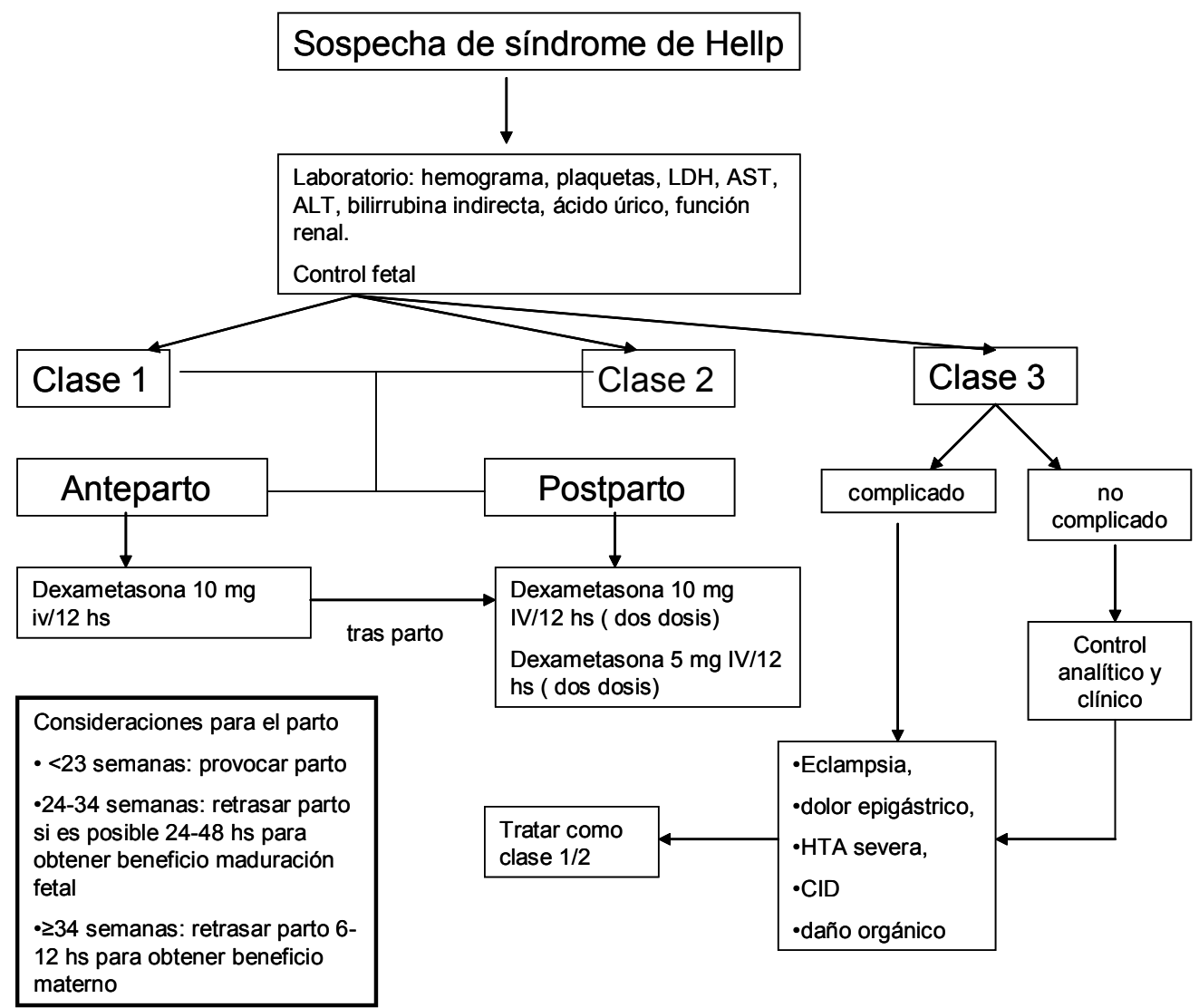

Figura 3.Corticoterapia en el Síndrome Hellp.

\section{VÍA DE PARTO}

La necesidad de adelantar el parto no obliga a la realización de cesárea de forma inmediata, y se pueden utilizar prostaglandinas para madurar el cérvix antes de inducción si el Bishop no es favorable, sin embargo debe evitarse una inducción prolongada ${ }^{18}$.

En el caso de gestaciones $<28$ semanas la tasa de éxito para parto vaginal se re- duce un $33 \%$ respecto a la semana 34 por mayor riesgo de pérdida de bienestar fetal y fracaso de inducción; de ahí que algunos autores recomienden cesárea para las gestantes con preeclampsia severa $<30$ semanas y Bishop bajo $<5$.

En caso de cesárea, la laparotomía media infraumbilical se asocia a menos complicaciones que la incisión de Pfannenstiel. No obstante, con recuento de plaquetas 
$>50.000 / \mu \mathrm{l}$ se puede optar por esta última incisión, se recomienda dejar un drenaje subaponeurótico, evitar la exploración del hígado por el riesgo de rotura de posibles hematomas subcapsulares y realizar profilaxis de la enfermedad tromboembólica con heparina de bajo peso molecular.

La anestesia locorregional consigue un buen control de la HTA y mejora el flujo uteroplacentario, por lo que es una técnica de elección en estas pacientes siempre que no existan contraindicaciones.

En el puerperio evitar la administración de metilergometrina para prevenir la hemorragia, porque aumenta la TA. En caso de hemorragia se puede utilizar tanto oxitocina como prostaglandinas.

\section{BIBLIOGRAFÍA}

1. Davey DA, MacGillivray I. The classification and definiion of the hypertensive disorders of pregnancy. Am J Obstet Gynecol 1988: 158892-158898.

2. Duley L, Henderson-Smart DJ, Meher S, King JF. Agentes antiplaquetarios para la prevención de la preeclampsia y de sus complicaciones (Revisión Cochrane traducida). En: La Biblioteca Cochrane Plus, 2008 Número 4. Oxford: Update Software Ltd.

3. Mires GJ, Williams FL, Leslie J, Howie PW. Assesment of uteral arterial notching as a screening test for averse pregnany outcome. Am J Obstet Gynecol 1998; 179: 1317-1323.

4. Cunningham FG, Lindheimer, MD. Hypertension in pregnancy. N Engl J Med 1992; 326 : 927-932.

5. Redman CW, SARgent IL. Latest advances in understanding preeclampsia. Science 2005; 308: 1592-1594.

6. Sibai B, Dekker G, Kupferminc M. Pre-eclampsia. Lancet 2005; 365: 785-789.
7. Duley L, Meher S, Abalos E. Management of pre-eclampsia. Brit Med J 2006; 332: 463-468.

8. RCOG. The management of severe preeclampsia, eclampsia. 2006. Guideline No. 10 (A).

9. SiBAI BM. Diagnosis and management of gestational hypertension and preeclampsia. Obstet Gynecol 2003; 102: 181-192.

10. The magpie trial. Collaboration Group. Do women with pre-eclampsia, and their babies, benefit from magnesium sulphate? The magpie Trial: a randomised placebo controlled trial. Lancet 2002; 359: 1877-1890.

11. SIBAI BM. The magpie trial. Lancet 2002; 360: 1329; author reply 1331-1132.

12. SiBAI BM. Diagnosis, prevention and management of eclampsia. Obstet Gynecol 2005; 105: 402-410.

13. Weinstein L. Syndrome of hemolysis, elevated liver enzymes, and low platelet count: a severe consequence of hypertension in pregnancy. Am J Obstet Gynecol 1982; 142: 159-167.

14. SiBAI BM. Diagnosis, controversies, and management of the syndrome of hemolysis, elevated liver enzymes and low platelet count. Obstet Gynecol 2004; 103: 981-991.

15. Martin JN, Thigpen BD, Rose CH, Cushman J, Moore A, MAY WL. Maternal benefit of high dose intravenous corticosteroid therapy for HELLP syndrome. Am J Obstet Gynecol 2003; 189: 830-834.

16. Magann EF, Bass D, Chauhan SP, Sullivan DL, Martin RW, Martin JN JR. Antepartum corticosteroids: disease stabilization in patients with HELLP syndrome. Am J Obstec Gynecol 1994; 171: 1148-1153.

17. James N. Martin JR. MD, Carl H. Rose, MD, ChrisTIAN M. BRIERY, MD. Understanding and managing HELLP syndrome: The integral role of agressive glucocorticoids for mother and child. Am J Obstet Gynecol 2006; 195, 914-934.

18. Nassar AH, Adra AM, Chakhtoura N, Gómez-MaRín O, Beydoum S. Severe preeclampsia remote from term: labor induction or elective cesarean delivery? Am J Obstet Gynecol 1998; 179: 1210-1213. 
